\title{
Ascending projections of the dorsal raphe nucleus to the caudate-putamen complex in rat: A degeneration and anterograde tracing study
}

\author{
Ali Jahanshahi*a,b,c Yasin Temel $^{\mathrm{a}, \mathrm{b}, \mathrm{c}}$, Harry W.M. Steinbusch ${ }^{\mathrm{a}, \mathrm{c}}$ \\ ${ }^{a}$ Departments of Neuroscience, Maastricht University, Medical Center, Maastricht, The Netherlands \\ ${ }^{b}$ Departments of Neurosurgery, Maastricht University, Medical Center, Maastricht, The Netherlands \\ ${ }^{c}$ European Graduate School of Neuroscience (EURON)
}

\begin{tabular}{|c|c|}
\hline ARTICLE INFO & ABSTRACT \\
\hline $\begin{array}{ll}\text { Article History } & \\
\text { Received } & 12 / 08 / 2012 \\
\text { Accepted } & 16 / 08 / 2012\end{array}$ & $\begin{array}{l}\text { Studies on neuropathological processes underlying the clinical syndromes of Parkinson's } \\
\text { disease (PD) have confirmed that PD affects both dopaminergic as well as serotonergic } \\
\text { projections of the dorsal raphe nucleus (DRN). These projections were investigated in }\end{array}$ \\
\hline $\begin{array}{l}\text { *Correspondence to: } \\
\text { Ali Jahanshahi } \\
\text { Department of Neuroscience, } \\
\text { Faculty of Health, } \\
\text { Medicine and Life Sciences, } \\
\text { Maastricht University, } \\
\text { UNS } 506229 \text { ER, Maastricht, } \\
\text { The Netherlands } \\
\text { e-mail: a.jahanshahianvar@np.unimaas.nl }\end{array}$ & $\begin{array}{l}\text { of the suggested mechanisms responsible for the progressive loss of dopamine (DA) } \\
\text { in PD is the oxidative stress-induced degeneration. The free radical nitric oxide (NO) } \\
\text { appears to be of special interest. The presence of this molecule in both the serotonergic } \\
\text { and dopaminergic DRN projections to the Caudate-Putamen (CPu) was examined in } \\
\text { the present study, using a combined neurochemical lesion technique and NADPH-d } \\
\text { histochemistry. NO and serotonin were found to be colocalized in the dorsomedial cluster } \\
\text { of the DRN. However, no NOS-immunohistochemistry was detected in the serotonergic } \\
\text { raphe-striatal projections. Loss of serotonergic neurons in the ventromedial dorsal raphe } \\
\text { resulted in a reduction of the striatal dopaminergic innervation. Degenerating serotonergic } \\
\text { elements were observed at both ends of the nigrostriatal pathway. Furthermore, loss of }\end{array}$ \\
\hline $\begin{array}{l}\text { Keywords: } \\
\text { Dorsal raphe nucleus } \\
\text { 5,7-Dihydroxytryptamine } \\
\text { 6-hydroxydopamine } \\
\text { Phaseolus vulgaris-leucoagglutinin } \\
\text { Tyrosine hydroxvlase }\end{array}$ & $\begin{array}{l}\text { degeneration of the raphe cells. Serotonergic raphe neurons may therefore be inhibited } \\
\text { by dopaminergic input of the DRN originating in the substantia nigra. Loss of this } \\
\text { inhibition might augment the hypothesized degeneration of the serotonergic raphe-striatal } \\
\text { projections in PD. Malfunction of such a feedback system could be a possible cause of } \\
\text { the DA deficiency in PD. } \\
\text { J. Exp. Clin. Med., 2012; 29:187-199 }\end{array}$ \\
\hline
\end{tabular}

Tyrosine hydroxylase

5-hydroxytryptamine

(C) 2012 OMU

\section{Introduction}

Idiopathic Parkinson's disease (PD) is a neurodegenerative disease affecting approximately $1 \%$ of the human population (Adams and Victor, 1993). It is principally characterized by tremor, rigidity, hypokinesia. Non-motor symptoms such as cognitive and affective symptoms are also commonly observed (Branchi et al., 2008).

The main pathological feature associated with $\mathrm{PD}$ is a marked loss of dopamine (DA) in the basal ganglia, as a result of degeneration of dopaminergic neurons in the substantia nigra, pars compacta (SNC) and ventral tegmental area of Tsai (VTA) (Hornykiewicz and Kish, 1987). Beside these, the dorsal raphe nucleus (DRN) is also known to contain dopaminergic neurons, which send efferent projections to the caudate-putamen (CPu) (Stratford and Wirtshafter, 1990). Degeneration of these dopaminergic neurons, and the consequent loss of their striatal projections, might contribute to the striatal DA levels.
Notwithstanding, in PD, the most dramatically changes occur in the dopaminergic system, significant changes have also been observed in several other neurotransmitter systems, e.g., the serotonergic system. Loss of serotonin has been reported in both DRN and striatum in PD (Chen et al., 1998).

Thus, both dopaminergic and serotonergic projections of the DRN are thought to participate in the onset and progress of PD. To investigate the exact role of these projections in this neurodegenerative disease, more anatomical data are warranted, i.e., data about origin, pathway and target areas. In the present study some anatomical aspects of the dopaminergic projection of the DRN to the $\mathrm{CPu}$ were investigated.

Dopaminergic projection of the dorsal raphe nucleus to the caudate-putamen

The DRN is a large, distinct cell mass situated in the tegmentum of the mesencephalon and pons. It can be divided in four subdivisions: A dorsomedial cluster, a ventromedial 
division, the lateral subnuclei (rostral subnucleus (B7)) and the caudal subnucleus (B6) (Steinbusch and Nieuwenhuys, 1982). The DRN compromises a large cluster of serotonin (5-hydroxytryptamine, 5HT)-containing nerve cell bodies in mammalian central nervous system (CNS) (Descarries et al., 1982). However, in addition to these serotonergic neurons, cells containing other transmitters have been demonstrated, e.g., DA (Trulson et al., 1985), $\gamma$-aminobutyric acid (Nanopoulos et al., 1982), enkephalin (Hokfelt et al., 1979) and substance P (Hokfelt et al., 1978) (Fig. 1).

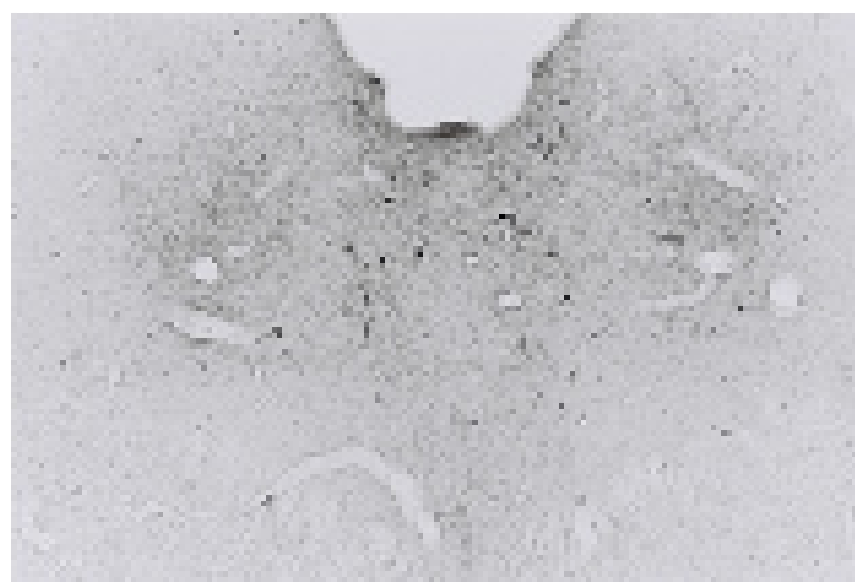

Fig. 1. The dorsal raphe nucleus as defined by its dopaminergic content at level A-760.

These neurons are known to innervate a great number of brain areas, e.g., the prefrontal cortex (Yoshida et al., 1989), substantia nigra (SN) and the striatum (Vertes, 1991). Besides these ascending pathways, there are descending projections from the dorsal raphe, i.e., to the locus coeruleus (LC) (Vertes and Kocsis, 1994). The afferents of the DRN have been shown to originate from a great number of brain areas, e.g., the $\mathrm{SN}$ and the lateral habenular nucleus.

Because it is harboring a large subset of neurotransmitters and it has a complex in and out put, the DRN is thought to participate in complex processes as sensory perception, mood and affect, feeding and meeting behavior, reward, sleep, aggression and cerebral blood flow (Mohammad-Zadeh et al., 2008). Because of its central role, it has also been implicated in a couple of neurodegenerative diseases, e.g., Alzheimer disease (Michelsen et al., 2008) and Parkinson's disease (Kaya et al., 2008).

In this study, the main focus will be on the dopaminergic neurons in the DRN and their projection to the $\mathrm{CPu}$. Reportedly, two primary subpopulations can be discriminated in the vicinity of the DRN. The first dopaminergic cell population (A10dc) is localized in the extreme dorsal border of the rostral half of the DRN and forms a thin band which borders the ventral surface of the cerebral aqueduct. The second, (A10c) occupies the medial aspect of the nucleus and extends in a dorsocaudal direction forms the ventrorostral border of the DRN where the cells appear contiguously with the dopaminergic neurons of the A10 group located in the caudal linear nucleus (CLi) (Stratford and Wirtshafter, 1990).

The population of the dopaminergic neurons was investigated in this report, is situated in the A10c group (Fig. 1).

This cell group extends rostrocaudally for a little more than $0.5 \mathrm{~mm}$, occupying the rostral third of this nucleus as de- fined by its content in 5HT neurons (Descarries et al., 1986). It begins rostral at the level of the oculomotor nucleus and reaches its greatest dimension at level A-770, and reduce abruptly at level A-470 (Steinbusch et al., 1980).

\section{Pathway of the ascending dopaminergic projection of the} dorsal raphe nucleus to the caudate-putamen

Little is known about the dopaminergic projection of these neurons in the DRN. Projections to the striatum, prefrontal cortex, nucleus accumbens (NAc) and lateral septum have been analyzed (Stratford and Wirtshafter, 1990). In the current report, attention has been focused on the first projection, i.e., the raphe-striatal pathway.

The pathway by which the dopaminergic neurons project upon the $\mathrm{CPu}$ is not easy to identify, because the dopaminergic neurons in the DRN are situated between the numerous other types of neurons (Descarries et al., 1986). So there are a couple of possibilities for these ascending projections, e.g., one of the major ascending serotonergic bundles or a VTAlike pathway. The ascending serotonergic fibers of the DRN consist of two bundles, known as the dorsal and ventral bundle, respectively. The fibers of the dorsal bundle mainly terminate in the caudal, ventrolateral division of the $\mathrm{CPu}$. Whereas, those of the ventral bundle, after having transverse the median forebrain bundle $(\mathrm{mfb})$, innervate a considerable number of the other telencephalic and diencephalic centers (Veening et al., 1980).

In the present study the ascending dopaminergic projection from the DRN to the $\mathrm{CPu}$ was examined using Phaseolus vulgaris-leucoagglutinin (PHA-L) (Vertes and Kocsis, 1994). This anterograde tracer was delivered in the ventromedial subdivision of the DRN, the location of the dopaminergic neurons.

\section{Efferents of the dorsal raphe nucleus in the caudate- putamen}

The dopaminergic innervation of the $\mathrm{CPu}$ has been studied extensively (Amalric and Koob, 1993; Joel and Weiner, 2000). However, little is known about the distribution distinct DRN derived dopaminergic terminals in the $\mathrm{CPu}$. The different parts of the $\mathrm{CPu}$ are related to the distinct function. Thus, whereas the dorsolateral $\mathrm{CPu}$ (non-limbic striatum) controls sensory motor function, the ventral part (limbic striatum) is implicated with affective behavior (Lynd-Balta and Haber, 1994). In PD, it is mainly the dorsal part which is affected (Graybiel et al., 1990). Because of this division, and its relation to PD, it is important to know where, and to what extend, the dopaminergic efferents of the DRN do terminate in the functional subdivision of the $\mathrm{CPu}$.

The DRN projects, through the major ascending bundle, to the caudoventral part of the $\mathrm{CPu}$. Reportedly, $80 \%$ of this projection is serotonergic and $20 \%$ is non serotonergic (Steinbusch et al., 1980). The non serotonergic component of this projection mainly compromises of dopaminergic cells (Descarries et al., 1986). It is uncertain however, as to whether this dopaminergic projection terminates either in the dorsal, ventral or the both parts of the $\mathrm{CPu}$ (Stratford and Wirtshafter, 1990) (Fig. 2). In this study the dopaminergic projection of the DRN was investigated at the level of the $\mathrm{CPu}$. Therefore, both the distribution of these dopaminergic fibers in the $\mathrm{CPu}$ and its contribution to the total dopamine innervation to of the 


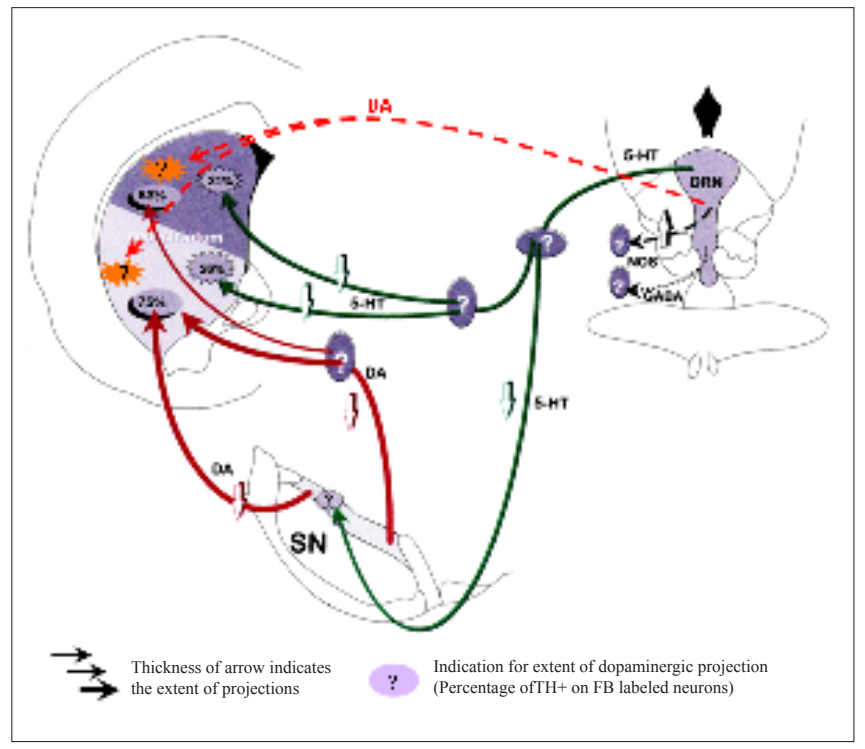

Fig. 2. Schematic representation of the interaction of the serotonergic and dopaminergic systems in dorsal raphe-nigro-striatal circuit in Parkinson's disease. DA, dopamine; DRN, dorsal raphe nucleus; GABA, $\gamma$-aminobutyric acid; NOS, nitric oxide synthase and 5HT, 5-hydroxytryptamine.

$\mathrm{CPu}$ were examined, using 6-hydroxydopamine (6-OHDA) and anterograde tracing techniques.

\section{Serotonergic projections of the dorsal raphe nucleus}

Serotonin inhibits dopaminergic neurons in the SN. It disrupts the physiological firing pattern of dopaminergic neurons and increase dopamine metabolism (Di Giovanni et al., 1999). In PD the serotonin level decreases, causing degeneration of these dopaminergic neurons and resulting in amelioration of the motor symptoms of PD (Hornykiewicz, 1998).

Since the serotonin content of the DRN is very high, it is not surprising that, in PD brains, a loss of serotonin has been detected in this nucleus. Besides, the existence of functional connection between serotonergic neurons of the DRN and the dopaminergic neurons of the nigro-striatal system has been suggested (Kelland et al., 1990).

To find evidence for such a functional connection, and to investigate the alleged role of the serotonergic projection of the DRN in PD, the neurotoxin 5,7-dihydroxytryptamine (5,7 DHT) was delivered to the ventromedial subdivision of the DRN, which is known to send a serotonergic projection to the $\mathrm{SN}$ and the striatum (Johnson and Ma, 1993). The effect of this selective serotonergic lesion on the dopaminergic innervation of the $\mathrm{CPu}$ was determined by $\mathrm{TH}$-immunohistochemistry. Functional connection was visualized by immunohistochemical double staining.

\section{Nitric oxide in the dorsal raphe nucleus}

One of the suggested mechanisms responsible for the progressive loss of dopamine in $\mathrm{PD}$ is the oxidative stress-induced degeneration (Emerit et al., 2004). The free radical nitric oxide (NO) appears to be of special interest since it has been implicated in the processes of neurodevelopment and neurotransmission. In brain tissue, NO can be produced by neuronal nitric oxide synthase (nNOS) containing cells (Yun et al., 1996). In the DRN, nNOS and 5HT coexist in neurons of the median subgroups (Steinbusch and de Vente, 1997), whereas nNOS and TH are separately described in midbrain nucleus (Johnson and Ma, 1993). Using selective neurotoxins and NADPH-d histochemistry, the presence of NO in the serotonergic and dopaminergic raphe-striatal projections was examined.

\section{Materials and methods Animals}

55 male albino Wistar rats, (weighing 240-260 gr) bred and housed at the Central Animal Facility of Maastricht University, Maastricht, The Netherlands) were used. They were housed in pairs in standard Makrolon ${ }^{\mathrm{TM}}$ cages on sawdust bedding in an air conditioned room (about $20^{\circ} \mathrm{C}$ ) under a 12-h reversed light/dark cycle (lights on from 17:00-05:00 h). Food, standard laboratory chow (Hopefarms, Woerden, the Netherlands) and water were available ad libitum. All experimental procedures were approved by the Animal Experiments and Ethics Committee of Maastricht University. The experiments were designed to minimize the number of animals used and their suffering.

\section{Surgery}

For surgical procedure animals were anaesthetized using a combination of ketamin and xylazine. In the first two parts of the study (A and B) the rats were positioned in a stereotactic apparatus. Subsequently a hole was drilled in the skull to expose the saggittal sinus. The zero of the lateral medial direction (LMe) was centered on this vein. Thereafter another hole was drilled in the skull following different LMe coordinates (rostrocaudal $(\mathrm{RC})=1.2 \mathrm{~mm}$, posterior to the ear bar (interaural). According to the dorsal-ventral coordinates (DV) and under the lateral approach of $32.5^{\circ}$, the injection needle or glass micropipette was lowered. All coordinates were according to the atlas of Paxinos and Watson (Paxinos and Watson, 1998).

\section{Perfusion and fixation}

After the survival time, which ranged between 0 and 14 days, the animal were anaesthetized with sodium pentobarbital and perfused in the ascending aorta with $200 \mathrm{ml} \mathrm{Ca}^{++}$free Tyrode's solution (1) or $200 \mathrm{ml} \mathrm{Krebs's} \mathrm{solution} \mathrm{(2).} \mathrm{These} \mathrm{solutions}$ were saturated with a mixture of $95 \% \mathrm{O}_{2}$ and $5 \% \mathrm{CO}_{2}$ gases, one hour prior to the perfusion at room temperature. This perfusion was followed by:

1) TH-, 5HT- and PHA-L-immunohistochemistry; $500 \mathrm{ml}$ Somogyi fixative containing 4\% paraformaldehyde (Merck), $15 \%$ picric acid (Sigma) and $0.05 \%$ glutaraldehyde (Merck) in $0.1 \mathrm{M}$ phosphate buffer (Baker; $\mathrm{pH}$ 7.6). Brains were removed and postfixed for $2 \mathrm{~h}$ in the same fixative omitting the glutaraldehyde. Thereafter brains were washed for $4 \mathrm{~h}$ in TBS at $4^{\circ} \mathrm{C}$.

2) DA-immunohistochemistry; $500 \mathrm{ml}$ glutaraldehyde fixative, containing $5 \%$ glutaraldehyde in $0.1 \mathrm{M}$ phosphate buffer ( $\mathrm{pH} 7.6)$ and ascorbic acid $(10 \mathrm{mM})$ were used. Brains were rapidly removed and postfixed for $30 \mathrm{~min}$ in the same fixative at $4^{\circ} \mathrm{C}$. They were washed for $4 \mathrm{~h}$ at $4^{\circ} \mathrm{C}$ in TBS, containing $10 \mathrm{mM}$ ascorbic acid.

Subsequently, the brains were cut serially on a vibratome into $50 \mu \mathrm{m}$ frontal sections and again stored at $4^{\circ} \mathrm{C}$. In some experiments (Determination of coordinates and 5HTimmunohistochemistry), cryostat section were cut, which were stored at $-20^{\circ} \mathrm{C}$. 


\section{(A) Neurochemical lesion study}

In the neurochemical lesion study 40 animal were divided into three groups:

(1) Determination of the stereotactic coordinates (Table 1).

(2) Determination of optimal coordination and volume of the 6-OHDA injection (Table 2).

(3) 6-OHDA, 5,7-DHT and combined (6-OHDA and 5,7DHT) lesion (Table 3).

All animals with the 6-OHDA and 5,7-DHT lesion of the DRN received desipramine (DMI; $20 \mathrm{mg} / \mathrm{kg}$ i.p, $1 \mathrm{~h}$ prior) to prevent any effect of toxins on noradrenergic neurons (Brezun and Daszuta, 2000). Rats were placed in a stereotactic apparatus (Stoelting, Wood Dale, USA; model 51653). After making burr holes in the skull, the rats received stereotactic $5 \mu \mathrm{l}$ injections of 6-OHDA or 5,7-DHT $(3 \mathrm{mg} / \mathrm{ml}$ dissolved in $0.9 \%$ saline and $0.2 \%$ ascorbic acid; Sigma, Zwijndrecht, The Netherlands) at DRN. Sham operated animals received saline in the same volume.

\section{(A1) Determination of stereotactic coordination}

The stereotactic coordinates of a microinjection in the ventromedial part of the DRN were estimated by means of ink injection. Different amount of ink and different DV coordinates were used (Table 1).

\section{Table 1. Determination of stereotactic coordinates}

\begin{tabular}{lcccc} 
Case & $\mathbf{D V}(\mathbf{m m})$ & $\mathbf{L M e}(\mathbf{m m})$ & $\mathbf{R C}(\mathbf{m m})$ & $\mathbf{I n k}(\boldsymbol{\mu l})$ \\
\hline Cases 1-3 & 5.9 & 0 & 1.2 & 0.2 \\
Cases 4-7 & 5.5 & 0 & 1.2 & 0.4 \\
Case 8 & 5.2 & 0 & 1.2 & 0.4 \\
Case 9 & 5.2 & -1.0 & 1.2 & 0.4 \\
Case 10 & 5.2 & 0 & 1.2 & $0.2^{*}$ \\
\hline
\end{tabular}

Bilateral injection $(* 0.2 \mu \mathrm{l}$ ink $)$

$\mathrm{DV}$, dorsal-ventral; LMe, lateral-medial and RC, rostral-caudal

In one rat (case 7) we used sharp instead of blunt injection needle, to check if the corpus callosum diverted the needle tract. The ink was delivered in $0.04 \mu \mathrm{l} / \mathrm{min}$ and the needle was left in situ for another $5 \mathrm{~min}$. Directly after the injection the brain was removed and cut in blocks, from one block (A-930A-430 mm) some $15-\mu \mathrm{m}$-thick transverse sections were cut on glass in a cryostat, to locate the injection site immediately. The second experiment of this neurochemical lesion study ("determination of the optimal volume and concentration of 6-OHDA injection") revealed that the injection were not located correctly. So, three other ink injections were made to reveal the correct coordinates. Two unilateral (cases 8 and 9), one in which the LMe coordinate was shifted (it was turned to $-1.0 \mathrm{~mm}$ instead of the zero), and one bilateral injection.

\section{(A2) determination of optimal volume and concentration of 6-OHDA lesion}

For the estimation of the optimal volume and concentra- tion of the 6-OHDA injections, two groups of 10 rats each were used. In the first group, different volumes of the same 6-OHDA concentration $(1.0 \mathrm{mg} / \mathrm{ml})$ were injected (Table 2 , left panel).

In the second group, injection of a constant volume (1.0 $\mu 1)$ containing different concentrations were delivered (Table 2 , right panel). For the above experiments the stereotactic coordinates as revealed in the first group of ink injection were used.

Tyrosine hydroxylase (TH)-immunohistochemistry: After the survival time of 8 days the brains were fixed and sectioned by vibratome. TH-immunohistochemistry was carried out using mouse anti-TH (Incaster; 1:2000) as primary antibody. After rinsing steps with Tris-buffered solution (TBS), TBS-Triton X-100 (Sigma) and TBS and incubation with the secondary antibody (Nordic; 1:100 goat anti-mouse biotin), the sections were incubated with mouse peroxidaseantiperoxidase (PAP) complex (Nordic/DAKO; 1:1000). To visualize the reaction product, the sections were incubated with 3,3-diaminobenzidine tetrahydrochloride (DAB; Sigma).

\section{(A3) 6-OHDA and/or 5,7-DHT lesions}

Five lesion of the ventromedial part of the DRN were made, using the stereotactic coordinates determined in the second group of the ink injection, whereas in cases 4 and $51.0 \mu 13.0$ $\mathrm{mg} / \mathrm{ml}$ 6-OHDA solution was delivered, two other rats (cases 2 and 3) were injected with $1.0 \mu$ of $3.0 \mathrm{mg} / \mathrm{ml} 5,7-D H T$ solution. One rat (case 1) received a sham injection (Table 3 ). After a survival time of 8 days, the brains were processed for TH-immunohistochemistry.

\section{HT-immunohistochemistry}

The brains of rats 1,2 , and 3 were also prepared for 5HTimmunohistochemistry. To visualize the 5HT neurons, the same immunohistochemical protocol was used, i.e. the PAP technique of Sternberger (Sternberger, 1979). The antibodies applied were rabbit anti-5HT (1:10000) (Steinbusch and Nieuwenhuys, 1982), goat anti rabbit IgG/Fc specific (Nordic; $1: 60)$ and rabbit PAP (DAKO; 1:600), respectively.

The result of this lesion experiment showed that the injection site was situated medially, but the solution diffused to the cerebral aqueduct (Aq). Therefore the LMe stereotactic coordinate was changed ( 2.5 to $-0.5 \mathrm{~mm})$ and instead of lowe-

\begin{tabular}{lcccccc} 
Table 3. Volumes, concentrations and stereotactic coordinates; \\
6-hydroxydopamine & (6-OHDA) & and/or & $5,7-$ \\
\multicolumn{7}{c}{ Dihydroxytryptamine (5,7-DHT) lesions } \\
Case & $\begin{array}{c}\text { Volume } \\
(\boldsymbol{\mu l})\end{array}$ & $\begin{array}{c}\text { Concentration } \\
(\mathbf{m g} / \mathbf{m l})\end{array}$ & $\begin{array}{c}\text { DV } \\
(\mathbf{m m})\end{array}$ & $\begin{array}{c}\mathbf{L M e} \\
\mathbf{( m m})\end{array}$ & $\begin{array}{c}\mathbf{R C} \\
(\mathbf{m m})\end{array}$ & $\begin{array}{c}\mathbf{D} \\
(\mathbf{m m})\end{array}$ \\
\hline Case 1 & 1.0 & Saline & 5.2 & -0.7 & 1.2 & 0 \\
Cases 2-5 & 1.0 & 3.0 & 5.2 & -0.7 & 1.2 & 0 \\
Cases 6-10* & 1.5 & 3.0 & 5.2 & -0.5 & 1.2 & -0.8 \\
\hline Case 8, combination of 6-OHDA and 5,7-DHT; D, depth \\
\hline
\end{tabular}

Table 2. Determination of the optimal volume and concentration of a 6-hydroxydopamine injection

\begin{tabular}{lcclcc} 
Case & Volume $(\boldsymbol{\mu l})$ & Concentration $(\mathbf{m g} / \mathbf{m l})$ & Case & Volume $(\boldsymbol{\mu l})$ & Concentration $(\mathbf{m g} / \mathbf{m l})$ \\
\hline Cases 1, 4, 7 & 2.0 & 1.0 & Cases 11, 14, 17 & 1.0 & 3.0 \\
Cases 2, 5, 8 & 1.0 & 1.0 & Cases 12, 15, 18 & 1.0 & 1.0 \\
Cases 3, 6,9 & 0.5 & 1.0 & Cases 13, 16, 19 & 1.0 & 0.33 \\
Case 10 & 2.0 & Saline & Case 20 & 1.0 & Saline \\
\hline
\end{tabular}


ring the needle to zero, it was lowered to $-0.8 \mathrm{~mm}(\mathrm{D}$, Table 3). It was decided to inject $1.5 \mu \mathrm{l}$ instead of $1.0 \mu 16$-OHDA solution, because the degenerated area was very small in rats 4 and 5. In order to be able to compare the results of this lesion experiment, we also delivered $1.5 \mu \mathrm{l}$ instead of $1.0 \mu \mathrm{l}$ 5,7-DHT.

Having changed both the LMe coordinate and injection size, five new injections were directed in the ventromedial part of the DRN. $1.5 \mu \mathrm{l}$ of $3.0 \mathrm{mg} / \mathrm{ml} 6-\mathrm{OHDA}$ or $5,7-\mathrm{DHT}$ was delivered in the brains of rats $6 / 9$ and rats $7 / 10$, respectively. Rat 8 received a combination of $1.5 \mu$ of $3.0 \mathrm{mg} / \mathrm{ml}$ 6-OHDA/5,7-DHT (Table 3). After 8 days the brains were processed for TH immunohistochemistry (all cases) or 5HTimmunohistochemistry (cases 7,8 and 10).

\section{Combined TH- and 5HT-immunohistochemistry}

Some sections of the brain of rat 8 were used for immunohistochemical double staining of both TH- and 5HTimmunoreactive elements, to visualize possible functional connections between dopaminergic and serotonergic neurons. TH- and 5HT-double labeling was carried out using mouse anti-TH (Incaster; 1:2000) and anti-5HT (1:10000) as primary antibody. After rinsing steps and incubation with the secondary antibodies (Nordic; 1:100 goat anti-mouse IgG/Fc and 1:60 goat anti-rabbit $\mathrm{IgG} / \mathrm{Fc}$ ), the sections were incubated with mouse PAP (De Jong et al., 1985). (Nordic/DAKO; 1:1000) subsequently they were incubated with DAB. After washing steps, the sections were incubated with rabbit anti$\mathrm{PAP}$ and DAB, respectively.

\section{(A4) Nitric oxide in the dorsal raphe nucleus and caudate- putamen}

In all five rats of the first lesion experiments (cases 1-5), nNOS was visualized in the DRN and CPu by NADPH-d histochemistry (Vincent and Kimura, 1992). After fixation and sectioning, one series of sections was washed in $50 \mathrm{mM}$ TrisHCL (Sigma; containing 0.2\% Triton X-100 PH 8.0) and incubated in $50 \mathrm{mM}$ Tris-HCL containing $0.42 \mathrm{mg} / \mathrm{ml}$ nitroblue tetrazolium (Boehringer) and $0.84 \mathrm{mg} / \mathrm{ml}$ (b-NADPH, Serva) at $37^{\circ} \mathrm{C}$ for $30-60 \mathrm{~min}$. Following the reaction, the sections were rinsed in $50 \mathrm{mM}$ Tris-HCL and mounted, dehydrated, cleared and cover slipped.

\section{(B) Anterograde tracing study}

To trace the connection between the ventromedial part of the DRN and the $\mathrm{CPu}$, the antrogradely transported tracer, PHA-L (Luiten et al., 1988) was injected into 15 animals by iontophoresis (Stoelting, Wood Dale IL; $7.5 \mu \mathrm{amps}$, pulsed at 7-second intervals for 10-13 minuets) through a stereotactically positioned glass micropipette (tip diameter $13 \mu \mathrm{m}$ ) into the target area, based on the coordinates of the atlas of Paxinos and Watson. Thereafter, the pipettes were left in-situ for 10 minutes.

(B1) determining the injection time of PHA-L injection

To determine the injection time of the PHA-L injection, in order to produce an injection site of the correct diameter, we delivered the anterograde tracer in the DRN of three rats.

PHA-L-immunohistochemistry

The injected and transported lectin was revealed by incubati- on of sections using the peroxidase-antiperoxidase method as described above. The following antibodies were applied; goat anti PHA-L (Vector; 1:4000), donkey anti goat (Nordic; 1:50) and goat-PAP (Nordic; 1: 500).

\section{(B2) PHA-L injections}

A positive current had to be delivered for 30 minutes to produce an injection of the intended size (diameter $500 \mu \mathrm{m}$ ). All injections were situated too low, i.e., in the median raphe nucleus (MnR). Therefore, the DV coordinate was changed (Table 4) and 6 new injections were made. The other parameters remained constant and after 13 days, the brains were fixed, sectioned and PHA-L-containing neurons were visualized as described above (Table 4).

\begin{tabular}{|c|c|c|c|c|}
\hline Case & DV (mm) & LMe (mm) & $\mathrm{RC}(\mathrm{mm})$ & D (mm) \\
\hline Cases 1, 2, 3 & 5.2 & -0.4 & 1.2 & -0.8 \\
\hline Cases 6, 9 & 5.0 & -0.4 & 1.2 & -0.8 \\
\hline Cases $4,7,13,14$ & 4.8 & -0.4 & 1.2 & -0.8 \\
\hline Cases $5,8,11,12,15$ & 4.6 & -0.4 & 1.2 & -0.8 \\
\hline Case 10 & 4.2 & -0.4 & 1.2 & -0.8 \\
\hline
\end{tabular}

Using the stereotactic coordinate $\mathrm{DV}=4.6$ or $4.8 \mathrm{~mm}$ the injection was located in the ventromedial part of the DRN. Five other rats were injected using these coordinates (Table 4).

Rat 10 was used to describe the major ascending bundle for the DRN. After 14 days the rat brains were processed for PHA-L-immunohistochemistry (all cases), TH- and 5HT-immunohistochemistry (cases 11 and 14) and NADP-dhistochemistry (cases 10 and 13). Following the injection, the dopaminergic nature of the PHA-L-containing fibers in the $\mathrm{CPu}$ had to be determined using fluorescence double staining. However, this technique was not applied, since no correctly located injections sites could be detected.

\section{Results}

Dopaminergic projection of the dorsal raphe to the caudate-putamen (I)

\section{a) Neurochemical lesion study}

Determination of stereotactic coordinates

The injected ink (cases 1-7, table 1) revealed, that for a lesion of the part of the DRN, the following coordinate should be used; DV: $5.2 \mathrm{~mm}$ and RC: $1.2 \mathrm{~mm}$ and a lateral approach of $32.5^{\circ}$. The second series of ink injection (cases 8-10, Table 1) revealed that, for a lesion of the ventromedial part of the DRN, the LMe coordinate had to be shifted, despite of earlier results. After having drilled a hole at the stereotactic coordinates RC: $1.2 \mathrm{~mm}$ and LMe: $2.8 \mathrm{~mm}$, the needle had to be shifted to LMe: -0.7 instead of the zero before it was lowered.

\section{Determination of optimal volume and concentration of 6-OHDA injection}

To determine the concentration and volume of a 6-OHDA injection in the ventromedial part of the DRN, consequently to produce an optimal lesion of dopaminergic neurons in this subnucleus, twenty rats were subjected to 6-OHDA injections in different volume and concentrations (Table 2). The injecti- 


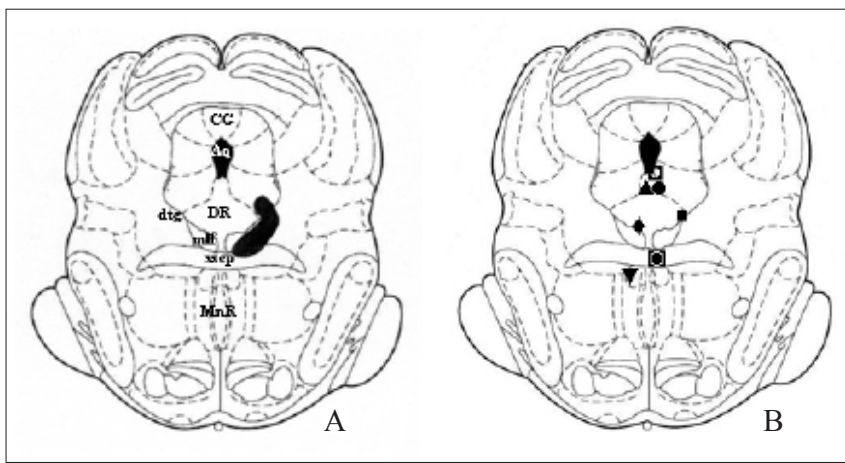

Fig. 3. Schematic representation of midbrain injections. Ten injections were made to find the optimal volume and concentration of a 6-hydroxydopamine injection in order to produce a lesion of the intended size, their location is shown in A. The locations of ten injections, in order to lesion the dorsal raphe, are shown in B. Aq, aqueduct; CG, central gray; DRN, dorsal raphe; dtg, dorsal tegmental nucleus; mlf, medial longitudinal fasiculus; MnR median raphe nucleus, and xscp, superior decussation of the cerebral peduncle. ( $\mathbf{\square}$ : case $1, \bullet$ : case $2, \boldsymbol{\Delta}$ : case 3 , $\mathbf{\square}$ : cases 4 and $5, \boldsymbol{\nabla}$ : case $6, \bullet$ : case 7 and $\mathbf{0}$ : cases 8,9 and 10 )

on sites were situated approximately $1 \mathrm{~mm}$ from the estimated position (Fig. 3A). Ipsilateral degeneration was detected in the dorsal tegmental bundle (dtg), retrorubral field, SN and the lateral VTA. The amount of degeneration observed was proportional to the volume and concentration of 6-OHDA. From these results we concluded that the most effective concentration of a 6-OHDA injection appeared to be $0.33 \mathrm{mg} / \mathrm{ml}$ in volume of 1.0 $\mathrm{ml}$. When using this amount, no damage was seen outside the injection site (Fig. $4 \mathrm{~A} / \mathrm{B})$.

\section{6-OHDA and/or 5,7-DHT lesions}

In the control group (cases 1, 19 and 20), rats received an injection of $1.0 \mu \mathrm{l}$ saline. Rat 1 , however, could not serve as control, due to severe bleeding in the region of injection, the sham injection caused much more degeneration that would be expected.

Another major problem was the location of the injection, as shown in figure $3 \mathrm{~B}$. Only a few injections were placed in, or nearby, the ventromedial part of the DRN. Although the different stereotactic coordinates were changed during the study (Table 1 and 3 ) only in one case (case 7) the neurotoxin could be delivered centrally in the ventromedial part of the DRN. Therefore, data from the different lesion studies were difficult to interpret.

\section{6-OHDA}

Rats 4 and 5 were injected with 6-OHDA, because both injections were situated in the near vicinity of the Aq (Fig. 3B), degeneration was found in its surroundings, even at large distance from the injection site. Damage in the dorsomedial subnucleus of the DRN was not detectible; probably because the neurotoxin diffused into the Aq. No degeneration in the ventral midbrain was observed.

The striata of the both rats showed a specific pattern of TH-staining. The dorsomedial and extreme dorsal parts (adjacent to the external capsule) of the $\mathrm{CPu}$ were stained very intensively. A very weak staining, interrupted with heavy spots, was found in the ventral and dorsolateral part of the $\mathrm{CPu}$. The

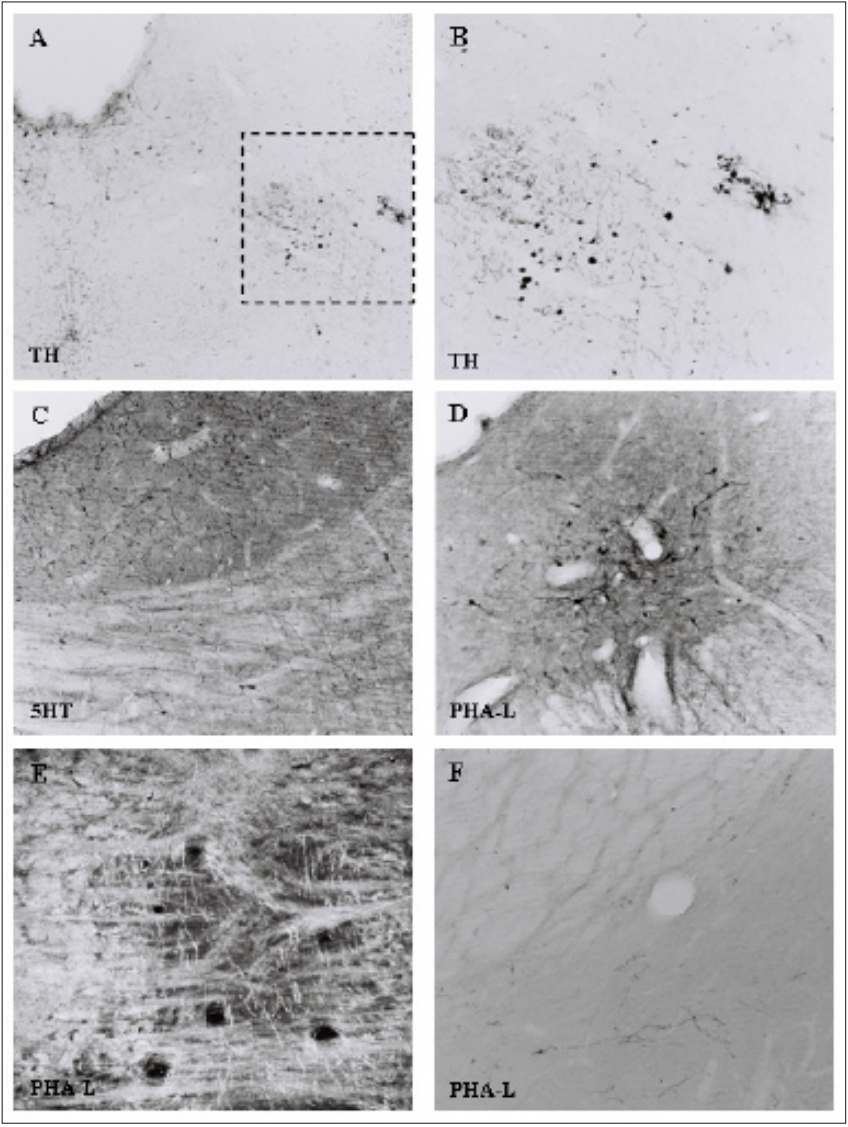

Fig. 4. To produce an optimal lesion of dopaminergic neurons in the ventromedial part of the dorsal raphe, twenty 6-hydroxydopamine (6-OHDA) injections were made. It appeared that the deliverance of $1.0 \mu \mathrm{l}, 3.0 \mathrm{mg} / \mathrm{ml}$ 6-OHDA resulted in a lesion of the intended diameter (A). A higher magnification of the inserted rectangle in A shows the degenerating dopaminergic cell bodies and fibers in the injection site (B). because the injections concerning cases 2-5 were situated near vicinity of the central aqueduct, the neurotoxin diffused into it and degeneration was found in its surroundings (C, 5HT/I-PAP staining). (D, E and F); Photomicrographs of PHA-L-containing neurons. PHA-L-containing fibers could be traced from the injection site (D), passing through the median forebrain bundle (E, dark-field) and the nucleus ansa lenticularis (F) to the caudate putamen (case 4). PHA-L, phaseolus vulgaris-leucoagglutinin; $\mathrm{TH}$, tyrosine hydroxylase ; and 5HT, 5-hydroxytryptamine.

$\mathrm{TH}$-staining of the $\mathrm{CPu}$ as observed in rat 1 showed a similar pattern of staining.

This aberrant staining of the $\mathrm{CPu}$ was merely due to a failure of the staining techniques, rather than to original differences in the dopamine innervation of the $\mathrm{CPu}$. This became evident when the $\mathrm{TH}$-immunoreactivity in the brains of rats 1 , 4 and 5 was compared to the TH-immunoreactivity in a wellstained case (case 20). In case of rats 1,4 and 5 most THimmunoreactive structures in the forebrain were not or very weakly visualized as compared to a normal TH-staining.

Having changed, both the stereotactic coordinates and the injection volume, two more rats were injected with 6-OHDA (cases 6 and 9). In both cases no detectible damage to the few $\mathrm{TH}$-immunoreactive structures present in the site of the injection was found. Neither was degeneration observed in other parts of the rat brain. 


\section{Serotonergic projection of the dorsal raphe nucleus}

\section{5,7-DHT}

After delivery of 5,7-DHT to the most dorsal part of the DRN of rats 2 and 3, most of the neurotoxin disappeared via the Aq, causing an extensive serotonergic degeneration in the central gray (CG), (Fig. 4D). Degeneration extended a few millimeters rostrally, and could even be observed in the dorsal part of the dorsomedial division of the DRN.

No loss of serotonin-immunoreactivity was seen in the $\mathrm{CPu}$ following lesioning of the DRN. The visualization of $\mathrm{TH}$-immunoractive elements in the $\mathrm{CPu}$ in these two cases, to trace possible interaction of the serotonergic and dopaminergic systems, showed the same problem as described above.

Having changed the stereotactic coordinates and injection volume, two more rats were injected with 5,7-DHT (cases 7 and 10). As shown in figure 3B (case 7) the injection was located centrally in the ventromedial subnucleus. This caused unilateral degeneration in the CG and a reduction of the number of serotonergic cell bodies in ventromedial subnucleus.

Furthermore degeneration was observed in the MnR, caudal linear nucleus (CLi), medial VTA and paramedian and caudal subnuclei of the interpeduncular nucleus (IPP and IPC, respectively). Also the medial part of the ventral ascending serotonergic bundle (VAB) was damaged at the level of the CLi and MnR.

In case 10 , the injection was situated as seen in figure 3B. At the level of the DRN, damage was observed in the ventral part of the ventromedial subnucleus. In addition severe degeneration was detected in CLi, MnR, medial VTA, medial SNC, IPP and IPC. Again the medial part of the VAB was damaged at the level of the CLi and MnR. Loss of 5HT-immunoractivity was observed in the caudal ventrolateral $\mathrm{CPu}$ after lesioning the ventromedial DRN. After THimmunohistochemistry, a decrease of TH-immunoreactive could be seen in the rostral dorsolateral $\mathrm{CPu}$.

\section{6-OHDA and 5,7-DHT}

Mixture of 6-OHDA and 5,7-DHT was delivered in medial part of the superior decussation of the cerebellar peduncle, (Figure 3B; case 8). Although severe damage to serotonergic neurons was observed in a number of areas, this injection did not cause any damage to either DRN, or in other part of the rat brain to dopaminergic neurons.

Degeneration accured in the ventromedial and lateral subnuclei of the DRN, CG, MnR, CLi, VTA, median parts of SNC, dorsal parts of median lemniscus (ml), IPP and IPC. As compared to case 10 (injection located at the same place), degeneration was more severe. In addition, a large reduction in the number of serotonergic cell bodies and fibers was observed, using the combination injection (6-OHDA and 5,7-DHT) rather than a single injection of 5,7-DHT. Many degenerating efferent were observed in the VAB. Compared to degeneration seen in this structure in case $10(5,7-\mathrm{DHT}$ lesion), the damage in rat 8 was more severe and extensive. The VAB was damaged not only medially, but degeneration also extended laterally at the level of the $\mathrm{MnR}$, in the reticular formation. The combined visualization of serotonergic and dopaminergic neurons in the DRN did not reveal any results.

\section{NO in the dorsal raphe nucleus}

In cases 2 and 3 (5,7-DHT lesion), a decrease of the number of NOS-immunoreactive neurons and processes was observed in the dorsomedial subnucleus of the DRN. This minor decrease was seen at the injection site only.

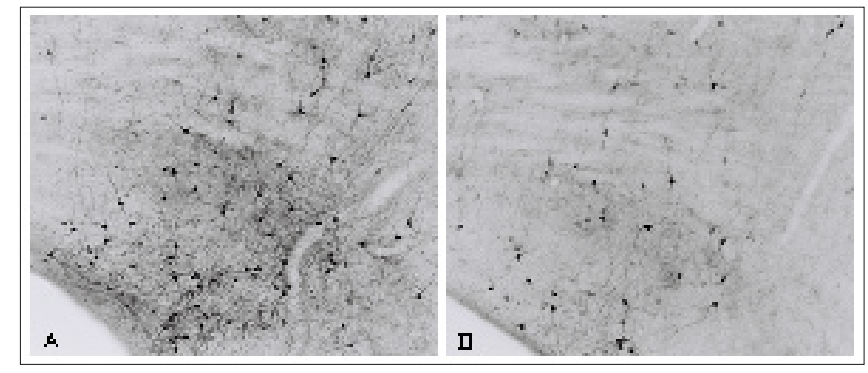

Fig. 5. Nitric oxide sythase (NOS)-immunoreactive cells and fibers in the central gray (CG). After delivery of 6-hydroxydopamine (6-OHDA) in the near vicinity of the central aqueduct, most of the neurotoxin diffused into the central aqueduct and caused degeneration in its surroundings. In the dorsolateral midbrain a loss of NOS-positive cells and fibers was detected after delivery of 6-OHDA (B) when compared to the non-lesioned (case 20) (A).

In case of 6-OHDA lesions (cases 4 and 5) the NOSimmunoreactivity had not changed at the level of the DRN. However, in both cases a considerable loss of NOSimmunoreactive neurons was observed in the dorsolateral midbrain, as compared to case 20 (Fig. 5).

None of the injection caused an alteration of the NOSimmunoreactivity at the level of the $\mathrm{CPu}$.

\section{Dopaminergic projection of the dorsal raphe nucleus to the caudate-putamen (II)}

b) Anterograde tracing

15 cases were injected with PHA-L (Table 4), changing the injection time in the first group (cases 1,2 and 3), it became evident that a positive current had to be delivered for $30 \mathrm{~min}$, in order to produce an injection site as large as the ventromedial part of the DRN. Again, the same problem as with the neurotoxin injections was encountered, i.e., the tracer had to be delivered centrally in the ventromedial subnucleus. So, stereotactic coordinates had to be changed following each group of injections (Table 4). Only in cases, in which afferents in the $\mathrm{CPu}$ could be traced will be reported here and are

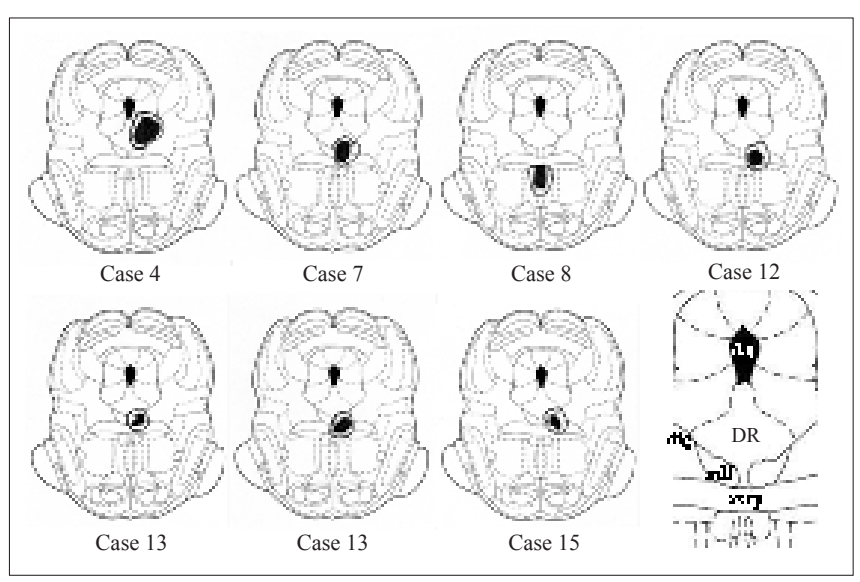

Fig. 6. Schematic representation of the PHA-L injection site. In cases 4, 7, 8, 13, 14 and 15 labeled was found in the caudate putamen. Black; heavy stained, uncolored; moderately stained. 
illustrated in figure 6 (Fig. 6).

In cases $4,7,8,12,13,14$, and 15, PHA-L containing fibers were observed in the $\mathrm{CPu}$.

In these cases, the labeled ascending fibers were followed, the location in which they entered the $\mathrm{CPu}$ was detected, and their distribution in this forebrain area was described. First, a general description of the pattern of projection, entrance of the $\mathrm{CPu}$ and distribution of the efferents is given, which is followed by a more specific description of each specific case.

(1) Projection, entrance and distribution; general pattern labeled fibers were found in multiple directions from the site of the injection. The two principal ascending routes were:

(1) (Ventro) Laterally: A group of axons passed (ventro) laterally from the injection site to the reticular formation and finally joined the $\mathrm{mfb}$.

(2) Ventrally: Another prominent group of fibers coursed ventrally to innervate several midbrain regions and finally joined the $\mathrm{mfb}$.

The lateral bundle of labeled axons coursed laterally from the site of the injection in the reticular formation and continued ventrally at a more rostral level (A-630), to pass round the red nucleus (RN) (A-560), few fibers left the bundle to innervate this nucleus. The labeled fibers shifted laterally dorsal to the dorsolateral part of the $\mathrm{ml}$ and extended to the SNC and in some cases to the SNr. The bundle of labeled fibers joined to the $\mathrm{mfb}$ at the caudal diencephalon (A-480).

The ventral bundle of these labeled axons coursed ventrally from the site of injection and passed laterally to the MnR and CLi (A-780-A-630). Some labeled fibers exited from this bundle to innervate both nuclei. This bundle of labeled fibers coursed rostrally, just dorsal/dorsolateral of the interpeduncular nuclei (IP), and medial part of the medial lemniscus. From this bundle fibers exited to innervate the dorsomedial $\mathrm{ml}$, VTA, SNC and IP (A-630-A-580). Further rostrally in the midbrain, the ventral bundle joined the $\mathrm{mfb}(\mathrm{A}-480)$. Fibers exited from the mfb to innervate the SNC and mamillary nucleus (A-480). Labeled axons exited laterally from the $\mathrm{mfb}$, first between the internal capsule (ic) and the $\mathrm{ml}$ (A-360) and more rostrally through the ansa lenticularis (al) (A-330-A160), to innervate the $\mathrm{GP}$ and $\mathrm{CPu}$. At this caudal level, most fibers passed the GP before innervating the ventromedial part of the ventral $\mathrm{CPu}$.

More rostrally (A-160-A060), this innervation appeared to be direct. Fibers existing from the mfb first passed the substantia innominata (SI) and the ventral pallidum (VP), before reaching the ventral $\mathrm{CPu}$. The most rostral labeled fibers (A-160-A-170), which innervated the $\mathrm{CPu}$, coursed ventral of the NAc to enter the ventrolateral $\mathrm{CPu}$, medial to the external capsule (ec). Only a few axons left this projection to terminate in the NAc.

In summary, coursing through the ventral or ventrolateral ascending bundle, labeled fibers terminated in several areas and finally joined the mfb. Axons left this bundle at several levels to innervate the $\mathrm{CPu}$. Caudally, this innervation was most intense and axons entered the ventrolateral $\mathrm{CPu}$ by passing the al and GP. More rostrally, axons exited from the $\mathrm{mfb}$, principally through the SI and VP, to moderately innervate the ventrolateral $\mathrm{CPu}$. At the most rostral level, fibers entered the ventrolateral $\mathrm{CPu}$, passing through the NAc. Although in most cases ipsilaterally ascending axons were mainly obser- ved, some contralaterally ascending fibers could be detected as well.

The distribution of labeled axons as observed in cases 4, $7,8,12,13,14$, and 15 at five different levels is summarized in figure 7 .

(I1) Distribution of dopaminergic fibers in caudate-putamen; specific pattern

In most cases, major or minor deviations from the general pattern were observed.

Case 4: Labeled fibers were observed at two levels in the $\mathrm{CPu}$, i.e., $\mathrm{A}-130$ and $\mathrm{A}-026$ :

A-130; labeled axons were observed both ipsilaterally and contralaterally in the $\mathrm{CPu}$. Ipsilaterally, a large number of fibers coursed through the ventral $\mathrm{CPu}$ and medially from the ec.

A-026; only ipsilaterally in the $\mathrm{CPu}$ fibers were observed, thus the dorsomedial, ventromedial and most ventrolateral parts.

Case 7: Labeled fibers were observed in the $\mathrm{CPu}$ at four levels:

A-130; extreme dorsally located fibers were observed.

A-026; some labeled axons terminated in the ventromedial $\mathrm{CPu}$.

A-100; few fibers distributed in the central part.

A-120; centrally located fibers project to the extreme ventromedial $\mathrm{CPu}$.

In this case, the neurons at the site of the injection were found to project completely ipsilateral to the $\mathrm{CPu}$.

Case 8: Labeled fibers were observed in the $\mathrm{CPu}$ at two levels:

A-230; labeled fibers were detected in the dorsomedial $\mathrm{CPu}$, adjacent to the LV.

A-130; a few fibers terminate in the extreme ventromedial part.

In case 8 , a moderate ipsilateral projection to the $\mathrm{CPu}$ was observed.

Case 12: A small number of labeled fibers were observed in the $\mathrm{CPu}$ at three levels:

A-130; few fibers were observed at the border of the dorsal and ventral $\mathrm{CPu}$.

A-026; and A 100; dorsally located fibers, adjacent to the ec. In case 12 a small ipsilateral projection to the $\mathrm{CPu}$ was observed.

Case 13: A large number of labeled fibers were observed in the $\mathrm{CPu}$ at four levels:

A-230; a few laterally located fibers adjacent to the ec, were observed. Contralaterally, a small number of medial located fibers were detected.

A-130; a small number of laterally orientated fibers was observed in the medial part of the $\mathrm{CPu}$, adjacent to the GP.

A-026; labeled axons coursed through the extreme ventromedial part of the $\mathrm{CPu}$. Another group of the fibers terminated at the border of the dorsal and ventral $\mathrm{CPu}$. Contralaterally, some ventromedially located fibers were observed.

A-100; ipsilaterally a large number of fibers was observed 
in the ventral part of the dorsal $\mathrm{CPu}$. Contralaterally, a small number of fibers were observed in the both dorsal and ventral $\mathrm{CPu}$.

Case 14: A moderate innervation of the $\mathrm{CPu}$ was observed at two levels:

$\mathrm{A}-230$ and $\mathrm{A}-026$; adjacent to the $\mathrm{CPu}$, a small number of laterally orientated fibers courses through the ventromedial $\mathrm{CPu}$. Another group of fibers coursed adjacent to the ec, towards the dorsal $\mathrm{CPu}$.

In case 14 an ipsilateral innervation of the $\mathrm{CPu}$ was observed.

Case 15: A relative large number of labeled fibers were observed at all five stereotactic levels in the $\mathrm{CPu}$ :

A-230; labeled fibers were observed to course through the extreme ventral $\mathrm{CPu}$.

A-130; ventrolaterally located fibers in the $\mathrm{CPu}$, adjacent to the ec, coursed dorsally.

A-026; fibers course extremely lateral, adjacent to the ec, to innervate the dorsal $\mathrm{CPu}$.

A-100; fibers were observed to terminate in the dorsal $\mathrm{CPu}$.

A-120; labeled fibers were observed in the extreme ventrolateral part of the $\mathrm{CPu}$, adjacent to the NAc.

In case 15 a mainly ipsilateral innervation of the $\mathrm{CPu}$ was observed.

No labeled fibers were found to innervate the $\mathrm{CPu}$ rostrally to the stereotactic levels A-040, A-180 A 100 and A 170 in cases 7, 12, 14 and 15, respectively, As well as to the level A-020 in cases 4,8 and 13 .

\section{Discussion}

\section{Dopaminergic projection of the dorsal raphe nucleus to} the caudate-putamen

To examine the serotonergic and dopaminergic raphe striatal projection, using the neurochemical lesion and anterograde tracing studies, either the neurotoxin or the anterograde tracer had to be delivered in the ventromedial subnucleus of the DRN.

However, throughout this study, the stereotactic coordinates had to be shifted to place the injection needle in the ventromedial subdivision of the DRN. After each series of injections, the DV-, LMe- and/or D-stereotactic coordinates were changed. Although most of these changes resulted in some of their intended effects, only the injection of the last PHA-L were situated approximately within or in the near vicinity of the ventromedial subnucleus.

Theoretically all injections according to DV: $5.5 \mathrm{~mm}$ and RC: 1.2 should be localized in the ventromedial subnucleus of the DRN, when using rats of 250 gr. However, most injections were situated in surrounding structures. Because the deviation was rather constant in each site of the injections (e.g., figure $3 \mathrm{~B}$ cases $2-5$ ) a systematic error had to be discovered somewhere in the application procedure.

Several possibilities were investigated. Using the blunt instead of sharp injection needle, did not result in correct located injection site, so it seems unlikely that the needle was diverted by brain structures, i.e., the corpus callosum. Alternatively the use of curved injection needle could have accounted for the observed differences in stereotactic coordinates. However, division of the injection was still seen when using straight glass pipettes.

- Pathway of the ascending dopaminergic projection of the dorsal raphe nucleus to the caudate-putamen

Numerous researchers (Steinbusch et al., 1980; Descarries et al., 1986; Stratford and Wirtshafter, 1990) have implicated possible pathways by which dopaminergic raphe striatal projection innervates the $\mathrm{CPu}$. In our study anterograde tracing study were used to find the route by which dopaminergic raphe neurons travel toward the $\mathrm{CPu}$. Concerning the anterograde tracing studies, PHA-L was delivered to the ventromedial DRN in one case only (figure 6, case 7). However in this experiment most of the PHA-L disappeared in the superior decussation of the cerebral peduncle (xscp).

Although no data were obtained about the pathway of the dopaminergic raphe-striatal projection, the projection pathway of the area bordering the ventromedial DRN could be determined.

The area containing the lateral and extremity ventral parts of the DRN, the dorsal MnR, the medial longitudinal fasciculus (mlf) and the xscp, respectively, was found to project to the $\mathrm{CPu}$ via a lateral and/or ventral pathway. Before joining the mfb fibers, the lateral bundle coursed through the reticular formation. The ventral bundle passed the CLi, MnR and VTA, to finally end up in the mfb. In the mfb both bundles were combined, innervating the $\mathrm{CPu}$ via the al or, more rostrally via the SI and VP. Laterally placed midbrain injections did project both contra- and ipsilaterally.

Distinct nuclei, confined to the area described above, were observed to contribute to the lateral and or ventral bundle. Further results of the anterograde tracing study will not be discussed here, because these data did not provide an answer to the solution of the hypotheses made in this study. However, these data may still be useful in further investigation of the projection patterns of distinct midbrain nuclei.

- Efferents of the dorsal raphe nucleus in the caudateputamen

In our hands, it appeared difficult to deliver either the neurotoxin or the anterograde neurotoxin to the ventromedial division of the DRN (figure 3B and 5, respectively). Thus only few suitable data were available.

\section{Distribution of the dopaminergic efferents of the dorsal raphe nucleus in the $\mathrm{CPu}$}

Although little PHA-L-immunoreactivity was detected in the $\mathrm{CPu}$ (Fig. 7), the projection area in the $\mathrm{CPu}$ arising from several areas bordering the DRN could be determined.

-The lateral subnucleus of the DRN (case 4) was found to innervate the caudal $\mathrm{CPu}$. This caudal innervation by lateral raphe cells is in agreement with previous studies of others (Pasquier et al., 1977; Steinbusch et al., 1980).

-The mediocaudal CPu was moderately innervated by the dorsal part of the MnR. Although this striatal projection was detected in other studies (Nicolaou et al., 1979), the distribution of these raphe efferents in the $\mathrm{CPu}$, is largely unknown.

-The area connecting the mlf, the most ventral part of the DRN and the xscp, was observed to innervate the ventral $\mathrm{CPu}$ at rostral levels. Thus, fibers coursed towards the dorsal $\mathrm{CPu}$ adjacent to the ec and GP to terminate in this part of the $\mathrm{CPu}$. This area is not known to project to the striatum.

The distribution of (dopaminergic) efferents of the DRN into the $\mathrm{CPu}$ may resemble one of the distribution patterns 


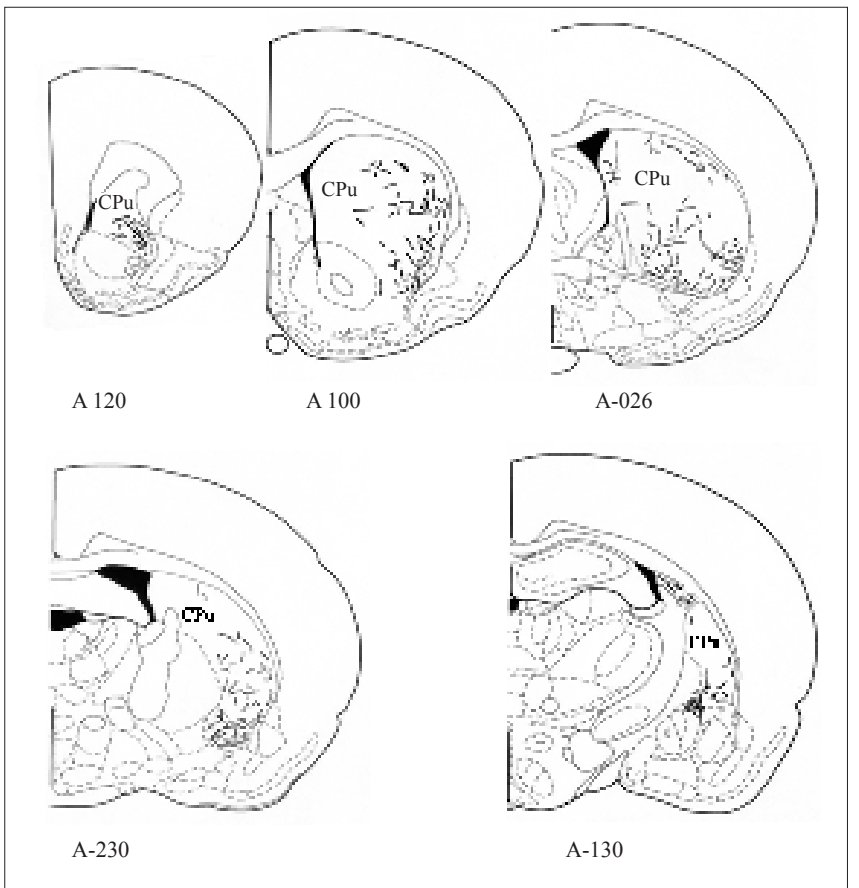

Fig. 7. Schematic representation of the labeling at five levels of the caudate-putamen produced by a total number of seven $\mathrm{PAH}-\mathrm{L}$ injections as shown in figure 6. $\mathrm{CPu}$, caudate-putamen.

described above or the general pattern (Fig. 7). However this has to be verified by further investigation.

\section{Contribution of the dopaminergic projection to the overall dopamine innervation of the caudate-putamen complex}

Our neurochemical lesion studies did not reveal data about the contribution of the dopaminergic raphe projection to the overall dopamine innervation of the $\mathrm{CPu}$.

The dopaminergic cell cluster of the DRN consists only of 1000-1500 cells, and is known to project to different areas (Trulson et al., 1985). These dopaminergic efferents might therefore be only responsible for a minor contribution to the total dopamine innervation of the $\mathrm{CPu}$. However, even malfunction of such a small projection might cause dramatic changes in the regulation of the striatal system. Further neurochemical lesions are needed to reveal the contribution of this raphe-striatal projection to the striatal DA levels.

\section{Serotonergic projection of the dorsal raphe nucleus}

The serotonergic efferents of the DRN are known to innervate $\mathrm{SN}$ and the $\mathrm{CPu}$ (van der Kooy and Hattori, 1980; Steinbusch, 1981). These raphe projections may therefore have a modulatory influence on the nigrostriatal dopaminergic pathway.

In the present study the serotonergic raphe input into the $\mathrm{SN}$ and the $\mathrm{CPu}$ could be verified by the selective neurochemical lesioning. Thus, after injection 5,7-DHT in the ventromedial DRN, degeneration was detected at both sites of the nigrostriatal pathway.

In concordance with a study of Veening (Veening et al., 1980) and Awouters (Awouters et al., 1990), the neurochemical lesion study showed that the ventromedial serotonergic raphe neurons innervate the caudal ventromedial $\mathrm{CPu}$. Degeneration of these serotonergic neurons caused a decrease of the $\mathrm{TH}$-immunoreactivity in the rostral dorsolateral $\mathrm{CPu}$.

A serotonergic raphe-derived modulatory influence on dopaminergic neurons has been described both in the SN and $\mathrm{CPu}$ (Awouters et al., 1990). In our study, the degeneration of serotonergic DRN neurons didn't result in decrease of the degree of $\mathrm{DA}$ innervation in the rostral dorsolateral $\mathrm{CPu}$, as estimated by $\mathrm{TH}$-immunoreactivity. These results can be explained by a direct striatal and/or an indirect influence, via the SN-VTA complex, of these serotonergic neurons.

Reportedly, the $\mathrm{CPu}$ receives a large amount of serotonergic afferents from the DRN (Steinbusch et al., 1981). Removal of this direct striatal input might result in reduction of the striatal DA levels, which is the main pathological feature associated with PD (Graybiel et al., 1990).

The precise nature of this serotonergic modulatory influence remains a topic for further investigation. Since in this study, the area of serotonergic degeneration was not observed the same part of the $\mathrm{CPu}$ as the decrease of the DA level, it is tempting to speculate that the serotonergic effect is mediated by a third type of neuron. Other neurotransmitter system, e.g., Cholinergic (Gillet et al., 1985) interneurons might be possible candidates to link these serotonergic and dopaminergic cell populations. Because in $\mathrm{PD}$ a reduction of the serotonin level of the CPu is observed (Chen et al., 1998), it will be important to further investigate the role of the direct raphe projection in this neurodegenerative disease (Gillet et al., 1985; Chen et al., 1998).

Another possible route of serotonergic influence on the $\mathrm{DA}$ level of the $\mathrm{CPu}$ is the nigrostriatal pathway. The DRN sends a large serotonergic projection to the SN (Imai et al., 1986). These serotonergic efferents have previously been shown to inhibit the dopaminergic neurons of this brain area ( Luiten et al., 1988; Kelland et al., 1990). Thus, removal of this inhibition might cause an increase in DA release in the SN and increase DA mediated inhibition of the firing rate of dopaminergic SN neurons. Thus, activation of DA autoreceptors inhibits the firing rate of neurons, resulting in a decrease in DA release from nigrostriatal DA fibers.

Furthermore, the ventromedial division of the SN-VTA complex is known to send its dopaminergic projections to the rostral dorsolateral CPu (Veening et al., 1980). A reduction of the DA level in the same area was found after lesioning the serotonergic raphe system. Thus, the decrease of the DA level of the $\mathrm{CPu}$, observed in this study, could be explained by the removal of the raphe serotonergic input of the $\mathrm{SN}$.

In $\mathrm{PD}$, the reduction of the $\mathrm{DA}$ level is mainly observed in the non-limbic striatum (dorsolateral $\mathrm{CPu}$ ) (Graybiel et al., 1990). In light of our results this reduction could, to some extend, be attributable to the disappearance of the serotonergic inhibition of dopaminergic SN neurons.

Some dorsal raphe cells are known to have collateral projections to both SN and CPu (Moukhles et al., 1997). Therefore, both a direct and indirect serotonergic influence might be responsible for the reduction of the striatal DA innervation.

Hypothetically, the degeneration of the raphe serotonergic neurons could be one of the major events in the onset of PD and could finally lead to deficiency in motor behavior as observed at earlier stages of PD, by reducing striatal DA levels.

Further investigation will be needed to verify the importance of the raphe serotonergic modulation of the dopaminergic neurons of the nigrostriatal pathway. More data are war- 
ranted, i.e., about the functional connections of both systems, the precise pathway of serotonergic modulation and the feedback loops of this system. Because the DRN is known to innervate a whole variety of different nuclei, and the $\mathrm{CPu}$ is innervated by even more brain areas, many pathways of (indirect) modulation may exist, which may equally contribute to the overall pathology of PD.

At the level of the DRN there is also evidence for interactions between the dopaminergic and serotonergic systems. A combined injection (6-OHDA and 5,7-DHT) showed to be more effective in damaging neurons than a single injection (5,7-DHT). Thus, the removal of dopaminergic neurons and fibers facilitates the degeneration of serotonergic neurons.

Little is shown about the functional connections between serotonergic and dopaminergic raphe system. However, serotonergic neurons in the DRN are inhibited by dopaminergic neurons in the SN (Martin-Ruiz et al., 2001). Following removal of this inhibition, the serotonergic neurons lose part of their control mechanisms and may be more sensitive to degeneration.

If degeneration of the serotonergic raphe system is one of the causes of PD, as hypothesized earlier, the loss of inhibition by dopaminergic SN cells of serotonergic raphe neurons may lead to an even higher loss of dopaminergic inhibition by an intensification of the initial serotonergic degeneration. Such a positive feedback loop could be responsible for the large reduction of the striatal DA levels. However, whether the onset of PD is accompanied by degeneration of either dopaminergic or serotonergic neurons, it is one of the main issues that remain to be solved. To solve this problem, data has to be obtained about the functional connection of both systems. Thus, do dopaminergic fibers terminate on serotonergic neurons or vice versa?

Besides modulation by nigral dopaminergic neurons, there could also be an effect of dopaminergic raphe cells on the serotonergic system of the DRN. Bi-directional interactions were not found in this study, since the removal of the serotonergic neurons did not affect dopaminergic degeneration in the DRN.

Further investigation is needed to create a clear view of the connection and feedback loops of the systems present in the DRN.

\section{Nitric oxide in the dorsal raphe nucleus}

Using NADPH-d histochemistry, we tried to detect NOimmunoreactivity in the serotonergic and dopaminergic raphe striatal projections. NOS-immunoreactivity diminished after 5,7-DHT injection in the dorsomedial subnucleus of the DRN. In line with other studies (Johnson and Ma, 1993) NOS and 5HT were shown to be colocalized in this midbrain area. No alteration of the appearance of the NO-system in the $\mathrm{CPu}$ was detected after lesioning the serotonergic raphe striatal projections. Thus, co-localization of 5HT and NOS could not be detected in these projections. However, in our studies the medial subnucleus DRN, which is known to project to the $\mathrm{CPu}$, had only been lesioned partially. Since the $\mathrm{CPu}$ receives a large serotonergic raphe input and $70 \%$ of the serotonergic raphe cells contain NOS (Wotherspoon et al., 1994), further investigation has to confirm our preliminary findings.

Following dopaminergic degeneration in the CG, a significant decrease of NOS-positive cells was detected in the dorsolateral midbrain. Since the dopaminergic cells are situated in this area, the uptake of the 6-OHDA by glial cells and consequent formation of free radicals and or free radical produced by degeneration of dopaminergic elements might be responsible for the loss of NOS-immunoreactivity. No further data was available about co-localization of $\mathrm{NO}$ and $\mathrm{TH}$.

\section{Conclusion remark}

Although the investigation of both the serotonergic raphe projection and the appearance of the NO-system revealed some suitable data, no anatomical data about the dopaminergic raphe striatal projection were obtained.

It appeared to be very difficult to deliver either the neurotoxin or the anterograde tracer to the ventromedial division of the DRN. In respect of the DRN this technical problem is observed in other works as well (Vertes and Kocsis, 1994).

Furthermore, once the solution has been delivered to the intended area, it might disappear into some surrounding structures, i.e., the Aq, the xscp or the mlf. Therefore, because of this contamination, data obtained from these cases were difficult to interpret.

Even when it becomes possible to deliver neurotoxin to the ventromedial cells, it will be hard to asses the proportion of the loss of these cells at the level of DA-immunoreactive varicose fibers. Only 1000-1500 dopaminergic neurons have been described in the DRN (Descarries et al., 1986), therefore, the DRN will contribute only to a minor extend to the dopaminergic innervation of the $\mathrm{CPu}$, as a result of overwhelming of TH-staining in this forebrain area. Moreover, due to this abundance of $\mathrm{TH}$-immunoreactive elements in the $\mathrm{CPu}$, it is also difficult to detect dopaminergic, PHA-L-containing fibers using fluorescence double staining in this area.

Another, maybe more applicable, way of gathering anatomical data about the dopaminergic raphe-striatal projection would be to combine retrograde tracing techniques and fluorescence double staining. Therefore, a small deposit of the retrograde tracer should be delivered to the whole extent of the $\mathrm{CPu}$. The retrograde tracer could be detected in dopaminergic cell bodies in the DRN, using fluorescence double staining techniques, to visualize both retrograde tracer and dopamine immunoreactive elements at the same time. Since little dopaminergic immunoreactivity is present in the DRN, this approach seems more promising.

\section{REFERENCES}

Adams, R.D., Victor, M., 1993. Priciples of neurology. New York: McGraw-Hill, 755-764.

Amalric, M., Koob, G.F., 1993. Functionally selective neurochemical afferents and efferents of the mesocorticolimbic and nigrostriatal dopamine system. Prog. Brain Res. 99, 209-226.

Awouters, F., Niemegeers, C.J., Megens, A.A., Janssen, P.A., 1990. Functional interaction between serotonin-S2 and dopamine-D2 neurotransmission as revealed by selective antagonism of hyper-reactivity to tryptamine and apomorphine. J. Pharmacol. Exp. Ther. 254, 945-951.

Branchi, I., D’Andrea, I., Armida, M., Cassano, T., Pezzola, A., Potenza, R.L., Morgese, M.G., Popoli, P., Alleva, E., 2008. Nonmotor symptoms in Parkinson's disease: Investigating early-phase onset of behavioral dysfunction in the 6-hydroxydopamine-lesioned rat model. J. Neurosci. 
Res. 86, 2050-2061.

Brezun, J.M., Daszuta, A., 2000. Serotonergic reinnervation reverses lesion-induced decreases in PSA-NCAM labeling and proliferation of hippocampal cells in adult rats. Hippocampus 10, 37-46.

Chen, C.P., Alder, J.T., Bray, L., Kingsbury, A.E., Francis, P.T., Foster, O.J., 1998. Post-synaptic 5-HT1A and 5-HT2A receptors are increased in Parkinson's disease neocortex. Ann. NY Acad. Sci. 861, 288-289.

De Jong, A.S., Van Kessel-van Vark, M., Raap, A.K., 1985. Sensitivity of various visualization methods for peroxidase and alkaline phosphatase activity in immunoenzyme histochemistry. Eur. J. Histochem. 17, 1119-1130.

Descarries, L., Berthelet, F., Garcia, S., Beaudet, A., 1986. Dopaminergic projection from nucleus raphe dorsalis to neostriatum in the rat. J. Comp. Neurol. 249, 511-520.

Descarries, L., Watkins, K.C., Garcia, S., Beaudet, A., 1982. The serotonin neurons in nucleus raphe dorsalis of adult rat: A light and electron microscope radioautographic study. J. Comp. Neurol. 207, 239-254.

Di Giovanni, G., De Deurwaerdere, P., Di Mascio, M., Di Matteo, V., Esposito, E., Spampinato, U., 1999. Selective blockade of serotonin-2C/2B receptors enhances mesolimbic and mesostriatal dopaminergic function: A combined in vivo electrophysiological and microdialysis study. Neuroscience. 91, 587-597.

Emerit, J., Edeas, M., Bricaire, F., 2004. Neurodegenerative diseases and oxidative stress. Biomed. Pharmacother. 58, $39-46$.

Gillet, G., Ammor, S., Fillion, G., 1985. Serotonin inhibits acetylcholine release from rat striatum slices: Evidence for a presynaptic receptormediated effect. J. Neurochem. 45, 1687-1691.

Graybiel, A.M., Hirsch, E.C., Agid, Y., 1990. The nigrostriatal system in Parkinson's disease. Adv. Neurol. 53, 17-29.

Hokfelt, T., Ljungdahl, A., Steinbusch, H., Verhofstad, A., Nilsson, G., Brodin, E., Pernow, B., Goldstein, M., 1978. Immunohistochemical evidence of substance P-like immunoreactivity in some 5-hydroxytryptamine-containing neurons in the rat central nervous system. Neuroscience. 3, 517-538.

Hokfelt, T., Terenius, L., Kuypers, H.G., Dann, O., 1979. Evidence for enkephalin immunoreactive neurons in the medulla oblongata projecting to the spinal cord. Neurosci. Lett. 14, 55-60.

Hornykiewicz, O., 1998. Biochemical aspects of Parkinson's disease. Neurology. 51, 2-9.

Hornykiewicz, O., Kish, S.J., 1987. Biochemical pathophysiology of Parkinson's disease. Adv. Neurol. 45, 19-34.

Imai, H., Steindler, D.A., Kitai, S.T., 1986. The organization of divergent axonal projections from the midbrain raphe nuclei in the rat. J. Comp. Neurol. 243, 363-380.

Joel, D., Weiner, I., 2000. The connections of the dopaminergic system with the striatum in rats and primates: An analysis with respect to the functional and compartmental organization of the striatum. Neuroscience. 96, 451-474.

Johnson, M.D., Ma, P.M., 1993. Localization of NADPH diaphorase activity in monoaminergic neurons of the rat brain. J. Comp. Neurol. 332 , 391-406.

Kaya, A.H., Vlamings, R., Tan, S., Lim, L.W., Magill, P.J., Steinbusch, H.W., Visser-Vandewalle, V., Sharp, T., Temel, Y., 2008. Increased electrical and metabolic activity in the dorsal raphe nucleus of Parkinsonian rats. Brain Res. 1221, 93-97.

Kelland, M.D., Freeman, A.S., Chiodo, L.A., 1990. Serotonergic afferent regulation of the basic physiology and pharmacological responsiveness of nigrostriatal dopamine neurons. J. Pharmacol. Exp. Ther. 253, 803-811.

Luiten, P.G., Wouterlood, F.G., Matsuyama, T., Strosberg, A.D., Buwalda, B., Gaykema, R.P., 1988. Immunocytochemical applications in neuroanatomy. Demonstration of connections, transmitters and receptors. Histochemistry. 90, 85-97.

Lynd-Balta, E., Haber, S.N., 1994. The organization of midbrain projections to the striatum in the primate: Sensorimotor-related striatum versus ventral striatum. Neuroscience. 59, 625-640.

Martin-Ruiz, R., Ugedo, L., Honrubia, M.A., Mengod, G., Artigas, F., 2001. Control of serotonergic neurons in rat brain by dopaminergic receptors outside the dorsal raphe nucleus. J. Neurochem. 77, 762-775.

Michelsen, K.A., Prickaerts, J., Steinbusch, H.W., 2008. The dorsal raphe nucleus and serotonin: Implications for neuroplasticity linked to major depression and Alzheimer's disease. Prog. Brain Res. 172, 233-264.

Mohammad-Zadeh, L.F., Moses, L., Gwaltney-Brant, S.M., 2008. Serotonin: A review. J. Vet. Pharmacol. Ther. 31, 187-199.

Moukhles, H., Bosler, O., Bolam, J.P., Vallee, A., Umbriaco, D., Geffard, M., Doucet, G., 1997. Quantitative and morphometric data indicate precise cellular interactions between serotonin terminals and postsynaptic targets in rat substantia nigra. Neuroscience. 76, 1159-1171.

Nanopoulos, D., Belin, M.F., Maitre, M., Vincendon, G., Pujol, J.F., 1982. Immunocytochemical evidence for the existence of GABAergic neurons in the nucleus raphe dorsalis. Possible existence of neurons containing serotonin and GABA. Brain Res. 232, 375-389.

Nicolaou, N.M., Garcia-Munoz, M., Arbuthnott, G.W., Eccleston, D., 1979. Interactions between serotonergic and dopaminergic systems in rat brain demonstrated by small unilateral lesions of the raphe nuclei. Eur. J. Pharmacol. 57, 295-305.

Pasquier, D.A., Kemper, T.L., Forbes, W.B., Morgane, P.J., 1977. Dorsal raphe, substantia nigra and locus coeruleus: Interconnections with each other and the neostriatum. Brain Res. Bull. 2, 323-339.

Paxinos, G., Watson, W., 1998. The rat brain in stereotactic coordinates. Australia: Academic Press.

Steinbusch, H.W., 1981. Distribution of serotonin-immunoreactivity in the central nervous system of the rat-cell bodies and terminals. Neuroscience. 6, 557-618.

Steinbusch, H.W., Nieuwenhuys, R., Verhofstad, A.A., Van der Kooy, D., 1981. The nucleus raphe dorsalis of the rat and its projection upon the caudatoputamen. A combined cytoarchitectonic, immunohistochemical and retrograde transport study. J. Physiol. 77, 157-174.

Steinbusch, H.W., van der Kooy, D., Verhofstad, A.A., Pellegrino, A., 1980. Serotonergic and non-serotonergic projections from the nucleus raphe dorsalis to the caudate-putamen complex in the rat, studied by a combined immunofluorescence and fluorescent retrograde axonal labeling technique. Neurosci. Lett. 19, 137-142.

Steinbusch, H.W.M., de Vente, J., 1997. New vistas on the neurobiology of depression: Colocalization of serotonin-, dopamine- and nitric oxide synthase-containing neurons in the dorsal raphe nucleus. In: Honig, A., van Praag, H.M., editors. Depression: Neurobiological, Psychopathological and Therapeutic Advances. New York: John Wiley. 179 -196.

Steinbusch, H.W.M., Nieuwenhuys, R., 1982. Localization of serotonin-like immunoreactivity in the central nervous system and pituitary of the rat, with special references to the innervation of the hypothalamus. In: Haber, B., Gabay, S., editors. Serotonin: Current Aspects of Neurochemistry and Function. New York: Plenum Press. 7-35.

Sternberger, L.A., 1979. Immunocytochemistry. In: Cohen, S., McCluskey, R.T., editors. Basic and clinical immunology. New York: John Wiley. 
Stratford, T.R., Wirtshafter, D., 1990. Ascending dopaminergic projections from the dorsal raphe nucleus in the rat. Brain Res. 511, $173-176$.

Trulson, M.E., Cannon, M.S., Raese, J.D., 1985. Identification of dopamine-containing cell bodies in the dorsal and median raphe nuclei of the rat brain using tyrosine hydroxylase immunochemistry. Brain Res. Bull. 15, 229-234.

van der Kooy, D., Hattori, T., 1980. Dorsal raphe cells with collateral projections to the caudate-putamen and substantia nigra: A fluorescent retrograde double labeling study in the rat. Brain Res. 186, 1-7.

Veening, J.G., Cornelissen, F.M., Lieven, P.A., 1980. The topical organization of the afferents to the caudatoputamen of the rat. A horseradish peroxidase study. Neuroscience. 5, 1253-1268.

Vertes, R.P., 1991. A PHA-L analysis of ascending projections of the dorsal raphe nucleus in the rat. J. Comp. Neurol. 313, 643-668.

Vertes, R.P., Kocsis, B., 1994. Projections of the dorsal raphe nucleus to the brainstem: PHA-L analysis in the rat. J. Comp. Neurol. $340,11-26$.

Vincent, S.R., Kimura, H., 1992. Histochemical mapping of nitric oxide synthase in the rat brain. Neuroscience. 46, 755-784.

Wotherspoon, G., Albert, M., Rattray, M., Priestley, J.V., 1994. Serotonin and NADPH-diaphorase in the dorsal raphe nucleus of the adult rat. Neurosci. Lett. 173, 31-36.

Yoshida, M., Shirouzu, M., Tanaka, M., Semba, K., Fibiger, H.C., 1989. Dopaminergic neurons in the nucleus raphe dorsalis innervate the prefrontal cortex in the rat: A combined retrograde tracing and immunohistochemical study using anti-dopamine serum. Brain Res. 496, 373-376.

Yun, H.Y., Dawson, V.L., Dawson, T.M., 1996. Neurobiology of nitric oxide. Crit. Rev. Neurobiol. 10, 291-316. 